\title{
Pure Ocular Myasthenia gravis
}

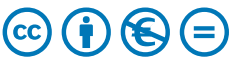

Authors

Lukas Kirzinger, Sophie Schötz, Berthold Schalke

\author{
Affiliation \\ Neurologische Klinik und Poliklinik der Universität \\ Regensburg im Medbo BKR, Germany \\ Key words \\ ocular myasthenia, synapse, antibodies \\ Bibliography \\ DOI https://doi.org/10.1055/s-0043-122245 \\ Neurology International Open 2018; 2: E51-E55 \\ (c) Georg Thieme Verlag KG Stuttgart · New York \\ ISSN 2511-1795 \\ Korrespondenzadresse \\ Prof. Dr. med. Berthold Schalke \\ Neurologische Klinik und Poliklinik der Universität \\ Regensburg im Medbo BKR \\ Universitätsstr 84 \\ 93053 Regensburg, Germany \\ berthold.schalke@medbo.de
}

\begin{abstract}
Pure ocular myasthenia was always described as a separate entity in historical publications. More than $50 \%$ of patients develop generalized symptoms in the course of the disease. Therefore pure ocular symptoms can be the beginning of generalized myasthenia, nevertheless there is a small group of patients who suffer lifelong purely ocular symptoms. The basis, therefore, seems to be special immunological, biochemical and pathophysiological mechanisms leading to specific local changes of the neuromuscular junction. The diagnostic procedure is hampered as antibodies against acethylcholine receptors and MuSK can be demonstrated in less than $50 \%$ of these patients. Therapy of pure ocular myasthenia does not differ fundamentally from other subgroups of myasthenia gravis and is implemented with cholinesterase inhibitors, steroids and common immunosuppressants. Thymomectomy is not recommended in general, but randomized controlled studies are lacking.
\end{abstract}

\section{Introduction}

Pure ocular myasthenia gravis (POMG), isolated over time, is a rare disease entity. On the other hand, the initial symptomology involving ptosis and double vision is the most common first clinical sign of myasthenia. This affects both the extraocular muscles (EOM), which are responsible for the formation of double vision and the levator muscles (LM), whose weakness leads to ptosis. Consequently more than $50 \%$ of POMG patients develop generalized myasthenia. For this reason, it is still debated today whether the POMG is an independent entity of myasthenia or whether it is only a milder clinical subgroup of generalized MG. Identification of antibodies is not always an aid in determining this. A portion ( $<50 \%)$ of POMG patients has antibodies against acetylcholine as well as more rare antibodies against muscle-specific kinase (MuSK). Among the remaining patients, typical immunoassays reveal no specific antibodies, i. e., more than $50 \%$ of POMG patients are seronegative. However, this only means that the disease-causing antibodies are undetectable with currently available assays [1-3]. Cell-based test procedures are required in this case, but they have not yet reached market maturity $[4,5]$.

A difficulty of exact classification is also the effect of a consistent initial treatment of primarily ocular symptoms, which may in- hibit the generalization of myasthenia gravis. Thus the composition of the group of POMG patients is heterogeneous, made up of patients with purely ocular myasthenia as well as those who would have developed generalized myasthenia if they had not been treated with immunosuppresive therapy [6].

\section{Case History and Clinical Symptoms of POMG}

In addition to ptosis, which is related to stress and which increases during the course of the day, POMG symptoms include double vision which varies in manifestation and intensity. Patients usually experience ptosis and double vision very early, but these symptoms do not always lead to immediate diagnostic clarification, as the patient's symptoms spontaneously recur due to fluctuation. In addition, since there is no pain, the symptoms are thus ascribed to non-specific reasons such as tiredness or general exhaustion. Ptosis can be unilateral or bilateral, symmetrical or asymmetrical, and, like double vision, can increase in bright sunlight, heat, or strenuous work, and can be relieved by wearing sunglasses or shutting the eyes temporarily. Heat increases the symptoms, whereas cold leads to improvement [7]. 
Eye movement disorders usually occur in combination with ptosis but may also be a separate symptom. There is, therefore, no typical pattern of ocular muscle involvement. However involvement of the medial rectus muscle with variable strabismus has been described. More rare are occurrences of isolated palsy of the inferior rectus or superior oblique muscles. Oculomotor nerve palsy can be imitated by the involvement of several eye muscles. In severely affected patients, complete external ophthalmoplegia may be apparent, which clinically cannot be distinguished from mitochondrial myopathies [8].

As the symptoms generally increase in intensity and duration over time, they ultimately require a medical examination, resulting in a Odyssey starting with the personal physician, cMRI and ophthalmologist and finally examination by a specialist. The duration from onset of the initial symptoms to a final diagnosis varies greatly, ranging from less than one day to many weeks/months, in some cases even years. A careful case history should always be obtained prior to a physical examination, since ptosis is actually a symptom commonly associated with other clinical syndromes, but is overlooked or misinterpreted and miscategorized by a non-neuromuscular ophthalmologist or neurologist.

The history should always determine whether the ptosis is congenital or acquired, whether other relatives also experience ptosis, whether symptoms increase with fatigue or physical stress, whether there has been past eye inflammation (Fuchs' blepharitis), and importantly, whether it has occurred simultaneously with double vision. Double images as a rule are suppressed in hereditary diseases and long-standing origins of ptosis and double visions, e. g. in the case of a deficit acquired in adolescence. Covering the pupil to block the visual field is not perceived. Blurred vision always occurs when the visual axes are no longer exactly aligned to each other, and is often the first and only long-perceived symptom before manifestation of double vision. Subsequently the images can no longer be exactly fused, resulting in the perception of double images. Occasionally double vision arises after successful treatment of the ptosis which initially obscured the eye. Frequently the patient finds this more disturbing that the ptosis itself.

\section{Clinical Diagnosis of POMG}

Special clinical scores used exclusively to determine or investigate POMG have not been established $[9,10]$; nowadays the testing of ocular symptoms is included in every myasthenia score [12]-[13]. Differentiated test methods for determining the extent of ptosis and double images such as Tensilon tonography, electronystagmography, and infrared oculography have shown limited usefulness for the assessment of POMG progression; they serve only to provide positive distinction between POMG and non-myasthenic causes of ptosis and double vision [14]. The most reliable test parameter for establishing the diagnosis of myasthenia is still antibody diagnosis, which is very often negative, especially in patients with pure POMG $[10,15]$. Seronegative does not mean that the patient does not possess antibodies against acetylcholine receptor structures, only that the antibodies cannot be determined with the usual test methods. An important clinical test with high specificity but moderate sensitivity (99\% / $75 \%$ [16]) is the so-called Cogan's lid twitch sign [17]. When the eyes are quickly moved to the prima- ry position by a rapid simultaneous movement after focusing on a fixed point, there is excessive upward movement of the upper lid/ lids and subsequent slow return drift of the eyelids to the ptotic position. This should be differentiated from typical blinking of the eyelids, which occurs always bilaterally and symmetrically. In very rare cases this can be caused by midbrain lesions [18], but these patients also have additional severe clinical neurological signs leading to a correct diagnosis. The Simpson test can verify a fatigue-related droop in the upper lid as well as double vision when looking upward. Analogously, the double-vision stress test can be applied in side view for at least $1 \mathrm{~min}$ [19]. The enhanced ptosis sign can be useful when examining patients with unilateral or asymmetrical bilateral ptosis. Manual raising of the more severely affected lid results in pronounced ptosis of the other side [20].

Patients perceive double vision as very disturbing, since spatial orientation can become almost impossible, and complain of blurry vision and dizziness; driving a vehicle or riding a bicycle is impossible. A significant problem for the patient is the frequently changing symptoms; initially patients try to overcome this by deliberately closing their eyes.

The red glass test can be useful, during which one eye is covered with a red filter and the patients is directed to look at an illuminated white circle. Normally only a pink circle is seen as a result of fusion of the red and white circle. In the case of double vision, this indicates exactly in which direction the double images appear and how far the two points of light (red and white) are from one another, and where they appear above or next to each other. This test is simple to perform and can be used before or after pharmacological testing or application of stress. If there are varying findings, then the diagnosis of myasthenia is largely established.

\section{Pharmacological Testing for POMG}

Pharmacological testing of ocular symptoms is highly sensitive, but unfortunately not specific. Either edrophonium chloride can be used, or, more easily, a $60 \mathrm{mg}$ tablet of pyridostigmine bromide can be administered. Patients with other neuromuscular diseases such as GBS, Lambert-Eaton syndrome or ocular myopathies can likewise demonstrate improvement of ocular symptoms. This must always be kept in mind when interpreting findings. The edrophonium chloride test should only be used in ultimately ambiguous cases, such as antibody-negative or electrophysiological negative testing, and when clinical findings are not definite. The procedure should always be performed by a person familiar with this procedure and carried out under appropriate safety conditions. Severe cardiac disease must be ruled out in advance, atropine must be available or even administered in advance. There must be sufficiently large intravenous access which is kept open by infusion throughout the procedure. One $\mathrm{ml}$ with $10 \mathrm{mg}$ of edrophonium chloride is thinned with $9 \mathrm{ml} 0.9 \%$ saline thus yielding a $1 \mathrm{mg} / \mathrm{ml}$ solution. The 1:10 diluted edrophonium chloride is administered intravenously; approx. $10-20 \%$ of the dose is initially applied. If the patient develops no clinical side effects, the remaining dose is injected within $30 \mathrm{~s}$ [21]. The neostigmine test can be performed as an alternative. After prior provocation of myasthenic weakness and subcutaneous injection of $0.5 \mathrm{mg}$ atropine, $1-2 \mathrm{mg}$ neostigmine $(1 \mathrm{mg} / 50 \mathrm{~kg}$ bodyweight) is injected intramuscularly. Effects should be expected 
after 5-10 min. This test can be combined simultaneously with repetitive stimulation in order to obtain an objective reading of the reduced drop in amplitude [22].

Administration of a $60 \mathrm{mg}$ pyridostigmine tablet is a safer but equally meaningful alternative. The test can be performed at any time in a neurological or general medical practice; ideally it should be accompanied by photographic documentation both prior to drug administration as well as an hour afterward.

A very simple procedure is the ice pack test [7] during which the palsied eyelid is chilled with a cold compress for $2 \mathrm{~min}$. Ptosis improves significantly in the case of ocular myasthenia, since acetylcholinesterase is inhibited at low temperatures. The test has high sensitivity, quite good specificity, is inexpensive, safe and rapid as well as feasible both as a bedside or office procedure.

\section{Electrophysiology}

Repetitive stimulation is a difficult examination to perform and interpret in cases of POMG. Since the relevant nerve/muscle pairs of the extraocular musculature and the levator muscle cannot be directly stimulated, as an alternative, the 7th cranial nerve is stimulated and derived at the orbicularis oculi. The decrement in amplitude is measured between the 1 st and 4 th or 5 th stimulus response. The text can be repeated after a functional stimulus. A $10 \%$ drop in amplitude is considered relevant, and in part the decrease in compound muscle action potential is considered even more sensitive. Technically, the problem is that the normal amplitude of the action potential in the facial musculature is already very low and very sensitive to stimulation artifacts $[23,24]$. A single-fiber EMG of the orbicularis oculi muscle can confirm the diagnosis if there is evidence of pathological jitter (and blockage) in several motor units.

\section{Testing for Antibodies}

As previously discussed, myasthenia-specific antibodies (AChR-ab, MuSK-ab) cannot be proven in many cases, therefore a seronegative finding does not rule out POMG. In the future, such cases should be tested for other known receptor structures such as LRP 4 , agrin or clustered acetylcholine receptors $[4,5,11,13,15]$.

\section{Differential Diagnosis of POMG}

The differential diagnosis of POMG is a wide field, with most diseases already ruled out by careful history and clinical examination. Today, a cranial MRI scan to rule out structural brain lesions, electrophysiological examination with repetitive irritation, and an EMG scan for evidence of myopathy are typically performed. In addition, an extensive laboratory investigation is performed. A proper diagnosis can frequently be ascertained based on clinical comorbidities such as pain resulting from diabetic ocular nerve palsy or unilateral pupil constriction resulting from Horner syndrome. Other important differential diagnoses include internuclear ophthalmoplegia (stroke / multiple sclerosis / tumor), involvement of the cranial nerves in cases of signs of poisoning, botulism, other neuromuscular diseases, endocrine orbitopathy or Miller-Fisher syndrome.

\section{Pathophysiological Considerations regarding POMG}

What explains the differences between POMG and generalized myasthenia? The extraocular musculature differs significantly in function from normal striated skeletal muscle. The extraocular musculature must accomplish precise control of the eyeballs in each of their respective optical planes. This is possible because these muscles have differing physiology. Fast fibers are responsible for the saccadic movements that position the eye exactly in the optical field. The slow tonic fibers are responsible for the fine adjustment of the eyeball. The receptor function, particularly in the slow fiber types, differs from normal skeletal muscles; the neuromuscular juncture is not as well developed as in the skeletal musculature. Acetylcholine receptor density is reduced and the synaptic folding is expanded with a consequently reduced safety factor compared to the skeletal musculature. The fast fibers in the extraocular musculature are similar to the structure of skeletal muscle receptors. These physiological anatomical differences may explain why extraocular musculature is more sensitive to disturbances $[25,26]$.

In addition, neuromuscular end plates of the extraocular muscles have two different subtypes of the acetylcholine receptor. 1.) Embryonic acetylcholine receptor with gamma subunits (found physiologically on embryonic muscle cells that are not yet innervated, as well as denervated muscle cells in adults, for example, in diabetic patients with severe polyneuropathy or on thymic muscle cells, which are also not innervated). 2.) Furthermore, normal adult ACHR with the alpha subunit located on all innervated muscle cells is found on the extraocular muscle. In the initial phase of myasthenia, when the immune cells in the thymus are activated or stimulated, the gamma subunit on the non-innervated thymus muscle cells is a possible antigen for increasing the myasthenic immune response. Possibly this is an explanation why ocular symptoms are so often the first clinical sign of myasthenia gravis [27]. Animal experiments have shown that immunological mechanisms, such as increased complement activation play a role [28]. Based on patients in whom conventional test methods have demonstrated no receptor-specific antibodies against the acetylcholine receptor, it has been shown that passive transmission of disease symptoms to mice is possible. This proves autoimmune pathogenesis.

\section{POMG Therapy}

In many cases, POMG is the first symptom of developing myasthenia. Consequently more than 50 \% of POMG patients develop generalized myasthenia, most within the first 2 years after onset. The goal of therapy is to prevent the widening of clinical symptoms if possible. Administration of prednisolone as well as azathioprine represents the first choice of immunosuppressive basic therapy. Alternatively, methotrexate, mycophenolate mofetil and tacrolimus can be used. During therapy escalation, rituximab or cyclosporin can be employed. Thus, the medication used is not different from typical therapeutic approaches to generalized myasthenia, followed by symptomatic therapy with cholinesterase inhibitors (pyridostigmine bromide). Currently only data from uncontrolled, 
non-randomized studies are available [29]. In part, purely symptomatic therapy suffices; these patients often do not require any immune-suppressing or modulating therapeutic measures in the further course of the disease. Especially with respect to POMG, however, there are patients who are hypersensitive to the administration of pyridostigmine and who exhibit strong side effects. There is evidence that consistent early treatment with prednisolone may delay and reduce the risk of subsequent generalization of POMG. In a retrospectively analyzed group of 87 patients with ocular myasthenia, 16 out of 32 patients treated only with pyridostigmine transitioned to generalized myasthenia within one year. On the other hand, this was the case for only 7 of 55 patients treated with prednisone. For patients treated with pyridostigmine only, this transition was on average after 0.2 years (range $0.1-0.8$ ), in those treated with prednisone, however, transition was only after 5.8 years (range $2.5-$ 10.5) [30].

Thymectomy seems to have no effect in patients with long-term pure ocular myasthenia. Nevertheless, all patients with POMG must be promptly screened for the presence of a thymoma or thymic hyperplasia visible on CT [31-34].

\section{SUMMARY}

POMG is often considered to be a less severe form of myasthenia, although it is a very debilitating disease, as it extends into many areas of a patient's life. Consistent treatment, both symptomatic and causal, allows the long-term prognosis of ocular myasthenia to be improved or the development of generalized myasthenia to be prevented. For patients with very pronounced purely ocular myasthenia who respond very poorly to various therapies, resorting to other aids such as attaching eyelid crutches to eyeglasses or eyelid surgery is certainly justified. As part of a long-term course with almost fixed double vision, a prismatic foil can also improve the symptoms. In this case, in close consultation with an ophthalmologist, a pragmatic approach should be suggested to the patient.

\section{Conflict of Interest}

The authors declare no conflicts of interest.

\section{References}

[1] Oosterhuis HJH. Myasthenia gravis. Groningen: Groningen Neurological Press; 1997

[2] Langmann A, Lindner S, Koch M et al. Okuläre Myasthenie: eine diagnostische und therapeutische Herausforderung - eine Übersicht. Klin Monatsbl Augenheilkd 2004; 22: 77-86

[3] Van den Berg B, Wohlgemuth M, Tijssen CC. Oculaire myasthenia gravis: Diagnose vaak vertraagd. Ned Tijdschr Geneeskd 2011; 155: A3241

[4] Gasperi C, Melms A, Schoser B et al. Anti-agrin autoantibodies in myasthenia gravis. Neurology 2014; 82: 1976-1983

[5] Pevzner A, Schoser B, Peters K et al. Anti-LRP4 autoantibodies in AChR- and MuSK-antibody-negative myasthenia gravis. J Neurol 2012; 259: 427-435
[6] Schumm F, Wiethölter H, Fateh-Moghadam A et al. Thymectomy in myasthenia with pure ocular symptoms. J Neurol Neurosurg Psychiatry 1985; 48: 332-337

[7] Kearsey C, Fernando P, D'Costa D et al. The use of the ice pack test in myasthenia gravis. JRSM Short Rep 2010; 1: 14

[8] Calvert CP. Disorders of neuromuscular transmission. In: Miller NR, Newman NJ, Biousse V, Kerrison JB, eds. Walsh \& Hoyt's clinical Neuro-Ophthalmology. 6th edition. Phliadelphia, Lippincott Williams \& Wilkins; 2005: 1041-1084

[9] Schumm F, Dichgans ]. Klinisches Bewertungssystem (Score) der oculären Symptomatik bei Myasthenia gravis. Nervenarzt 1985; 56: 186-187

[10] Barton J]. Quantitative ocular tests for myasthenia gravis: a comparative review with detection theory analysis. J Neurol Sci 1998; 155: 104-114

[11] Besinger UA, Toyka KV, Hömberg M et al. A. Myasthenia gravis: Long-term correlation of binding and bungarotoxin blocking antibodies against acetylcholine receptors with changes in disease severity. Neurology 1983; 33: 1316-1321

[12] Gajdos P, Sharshar T, Chevret S. Standards of measurements in myasthenia gravis. Ann N Y Acad Sc 2003; 998: 445-452

[13] Barnett C, Katzberg H, Nabav M et al. The quantitative myasthenia gravis score: comparison with clinical, electrophysiological, and laboratory markers. J Clin Neuromuscul Dis 2012; 13: 201-205

[14] Campbell MJ, Simpson E, Crombie AL et al. Ocular myasthenia: evaluation of tensilon tonography and electronystagmography as diagnostic tests. J Neurol Neurosurg Psychiatry 1970; 33: 639-646

[15] Oda K, Ito Y. Myasthenia gravis: Antibodies to acetylcholine receptor in ocular myasthenia gravis. J. Neurol. 1981; 225: 251-258

[16] Singman EL, Matta NS, Silbert DI. Use of the Cogan lid twitch to identify myasthenia gravis. J Neuroophthalmol 2011; 31: 239-240

[17] Cogan DG. Myasthenia gravis. A review of the disease and a description of lid twitch as a characteristic sign. Arch. Opthalmol 1965; 74: 217-221

[18] Ragge ML, Hoyt WF. Midbrain myasthenia, fatigable ptosis, ’lid twitch'sign, and ophthalmoparesis from a dorsal midbrain glioma. Neurology 1992; 42: 917-919

[19] Schneider-Gold C, Toyka KV. Myasthenia gravis Pathogenese und Immuntherapie Dtsch Arztebl. 2007; 104: A-420 / B-368 / C-356

[20] Gorelick PB, Rosenberg M, Pagano R]. Enhanced ptosis in myasthenia gravis. Arch Neurol 1981; 38: 531

[21] Dijk JL, Florence L. The tensilon test: A safe office procedure. Ophthalmology 1980; 87: 210

[22] Fox JM et al. Neurologische Pharmakotherapie. Stuttgart: Thieme Verlag; 2004: 217

[23] AAEM Quality Assurance Committee. American Association of Electrodiagnostic Medicine. Practice parameter for repetitive nerve stimulation and single fiber EMG evaluation of adults with suspected myasthenia gravis or Lambert-Eaton myasthenic syndrome: summary statement. Muscle Nerve 2001; 24: 1236-1238

[24] Katirji B. Electrodiagnosis of neuromuscular junction disorders. In: Kaminski HJ. ed. Myasthenia gravis and related disorders. Totowa, NJ, US: Humana Press; 2003: 149-175

[25] Serra A, Ruff RL, Leigh RJ. Neuromuscular transmission failure in myasthenia gravis: Decrement of safety factor and susceptibility of extraocular muscles. Ann N Y Acad Sci 2012; 1275: 129-135

[26] Liu R, Xu H, Wang G et al. Extraocular muscle characteristics related to myasthenia gravis susceptibility. PLoS One 2013; 8: e 55611

[27] Wekerle H, Ketelsen UP, Zurn AD et al. Intrathymic pathogenesis of myasthenia gravis: Transient expression of acetylcholine receptors on thymus-derived myogenic cells. Eur J Immunol 1978; 8: 579-582

[28] Wu X, Tuzun E, Li J et al Ocular and generalized myasthenia gravis induced by human acetylcholine receptor $\gamma$ subunit immunisation. Muscle Nerve 2012; 45: 209-216 
[29] Benatar M, Kaminski H. Medical and surgical treatment for ocular myasthenia. Cochrane Database Syst Rev 2012; 12: CD005081

[30] Kupersmith M]. Ocular myasthenia gravis: Treatment successes and failures in patients with long-term follow-up. J Neurol 2009; 256: $1314-1320$

[31] Kupersmith M], Ying G. Ocular motor dysfunction and ptosis in ocular myasthenia gravis: Effects of treatment. Br J Ophthalmol 2005; 89: $1330-1334$
[32] Hoffmann S, Kohler S, Ziegler A et al. Glucocorticoids in myasthenia gravis - if, when, how, and how much? Acta Neurol Scand 2014; 130 : 211-221

[33] Zach H, Cetin H, Hilger E et al. The effect of early prednisolone treatment on generalization rate in ocular myasthenia gravis. Eur J Neurol 2013; 20: 708-713

[34] Wong SH, Huda S, Vincent A et al. Ocular myasthenia gravis: Controversies and updates. Curr Neurol Neurosci Rep 2014; 14: 421 